\title{
Effets du travail du sol et de la fertilisation organo-minérale sur les rendements du mil en zone soudano-sahélienne du Burkina Faso
}

\author{
Siébou PALE ${ }^{*}$, Albert BARRO ${ }^{1}$, Mahamoudou KOUMBEM ${ }^{2}$, Aïda $_{\text {SERE }}^{2}$ et \\ Hamidou TRAORE ${ }^{1}$
}

\author{
${ }^{1}$ Intitut de l'Environnement et de Recherches Agricoles, 04 B.P 8645 Ouagadougou 04, Burkina Faso. \\ ${ }^{2}$ Université Joseph Ki-Zerbo, 03 B.P 7021 Ouagadougou 03, Burkina Faso. \\ *Auteur correspondant ; E-mail : siebout.pale@yahoo.fr; Tel. : +226 70331164 ; Fax : (226) 50340271
}

Received: 21-01-2021 $\quad$ Accepted: 25-04-2021 $\quad$ Published: 30-04-2021

\section{RESUME}

Le mil est la deuxième importante céréale cultivée au Burkina Faso après le sorgho. Ces dernières années, les rendements sont en baisse du fait de la pauvreté des sols et des aléas climatiques. Cette étude réalisée en 2018 et 2019 à la Station de Recherches de Saria avait pour objectif d'identifier des technologies de gestion de l'eau et de la fertilisation qui optimisent les rendements du mil. Le dispositif expérimental était un bloc complétement randomisé avec une disposition des traitements en split-plot et trois répétitions où les parcelles principales correspondaient à quatre niveaux de travail du sol et les parcelles secondaires à huit niveaux de fertilisation minérale avec ou sans compost. Les résultats ont montré que l'utilisation du compost plus les engrais minéraux ont permis des gains de 338 à $502 \mathrm{~kg} \mathrm{ha}^{-1}$ pour le grain et de 657 à $947 \mathrm{~kg} \mathrm{ha}^{-1}$ pour la paille. Le labour a engendré des gains de 266 à $635 \mathrm{~kg} \mathrm{ha}^{-1}$ pour le grain et de 381 à $601 \mathrm{~kg} \mathrm{ha}^{-1}$ pour la paille. Ces résultats permettent de recommander le labour avec une fertilisation organique et minérale pour optimiser les rendements du mil dans la zone soudano-sahélienne du Burkina Faso.

() 2021 International Formulae Group. All rights reserved.

Mots clés : Labour, zaï manuel, billonnage cloisonné, compost, Station de Recherches de Saria.

\section{Effects of tillage and organo-mineral fertilization on yields of pearl millet in the Soudano-sahelian zone of Burkina Faso}

\begin{abstract}
Pearl millet is the second important cereal crop cultivated in Burkina Faso after sorghum [Sorghum bicolor (L.) Moench]. In recent years, farmers failed to achieve good yields because of low productivity resulting from soil poverty and climatic risks. This study was carried out at Saria Research Station in 2018 and 2019 and aimed at finding the best combination of water management techniques and fertilizers to optimize pearl millet yields. The experiment was conducted in randomized complete block design with split plot arrangements of treatments and three replications. The main plot was tillage method with four levels and the sub-plot was fertilization with eight levels consisting of mineral fertilizers with or without compost. Results showed that the addition of compost to mineral fertilizers resulted in yield increases from 338 to $502 \mathrm{~kg} \mathrm{ha}^{-1}$ for grain and 657 to $947 \mathrm{~kg} \mathrm{ha}^{-1}$ for stover. The use of plough increased grain yield from 266 to $635 \mathrm{~kg} \mathrm{ha}^{-1}$ and stover from 381 to
\end{abstract}


$601 \mathrm{~kg} \mathrm{ha}^{-1}$. These results indicate that the use of compost plus mineral fertilizers and plough that optimize pearl millet yields can be recommended for pearl millet production in the Sudano-sahelian zone of Burkina Faso. (C) 2021 International Formulae Group. All rights reserved.

Keywords: Plough, manual zaï, tied-ridging, compost, Saria Research Station.

\section{INTRODUCTION}

Dans les pays sahéliens en général et au Burkina Faso en particulier, la faible productivité du secteur agricole est liée d'une part à la pauvreté naturelle des sols en matières organiques mais aussi en éléments nutritifs essentiels comme l'azote $(\mathrm{N})$ et le phosphore (P) (Dabré et al., 2016; Kohio et al., 2017), et d'autre part aux aléas climatiques notamment les sècheresses et l'inégale répartition des pluies (Diouf, 2001 ; Traoré, 2009 ; Nacro et al., 2010). L'agriculture burkinabè occupe $84 \%$ de la population active et contribue à $35 \%$ au PIB (MAAH, 2017a). Elle est donc un important secteur du développement du pays. En effet, les productions agricoles constituent la principale source de revenu des populations rurales burkinabè et procurent l'essentiel des besoins nutritionnels de la population (MAAH, 2017a). Cette agriculture qui reste toujours extensive, caractérisée par de petites exploitations familiales (MAAH, 2017b), fait face à de nombreuses contraintes limitant sa productivité.

Le mil [Pennisetum glaucum (L.) R. $\mathrm{Br}$.] qui constitue la deuxième céréale cultivée au Burkina Faso après le sorgho [Sorghum bicolor (L.) Moench] (MAAH, 2017a) est très ancré dans les habitudes alimentaires des populations (Békoye, 2011). La superficie des terres emblavées en mil pour la campagne agricole 2016/2017 a été de 1,2 millions hectares avec une production de 905 mille tonnes (MAAH, 2017a). Pour cette même campagne agricole, les rendements du mil étaient de $605 \mathrm{~kg} \mathrm{ha}^{-1}$ en Zone sahélienne, 603 $\mathrm{kg} \mathrm{ha}^{-1}$ en zone soudano-sahélienne et $692 \mathrm{~kg}$ $\mathrm{ha}^{-1}$ en Zone soudanienne (MAAH, 2017a). Quant au sorgho, il a connu des rendements de $697 \mathrm{~kg} \mathrm{ha}^{-1}$ en Zone sahélienne, $888 \mathrm{~kg} \mathrm{ha}^{-1}$ en zone soudano-sahélienne et $1079 \mathrm{~kg} \mathrm{ha}^{-1}$ en Zone soudanienne. Malgré les actions multiformes entreprises par la recherche, les producteurs, les ministères en charge du développement agricole et les décideurs politiques pour accroitre la productivité agricole, l'agriculture burkinabè reste extensive avec une production de mil insuffisante pour satisfaire les besoins alimentaires des populations (Zougmoré, 2003 ; Hien, 2004 ; Palé et al., 2009 ; Zongo, 2013 ; Sermé, 2014 ; Dabré et al., 2016 ; Kohio et al., 2017).

L'objectif de la présente investigation est d'identifier des technologies de gestion de l'eau et de la fertilisation organo-minérale qui permettent d'optimiser le rendement du mil. Les technologies prometteuses issues de cette étude seront mises à la disposition des producteurs pour leur permettre d'optimiser la production de mil tout en améliorant les revenus de ces producteurs.

\section{MATERIEL ET METHODES Site de l'étude}

L'étude a été conduite en 2018 et 2019 à la Station de Recherches Environnementales et Agricoles de Saria au Burkina Faaso $\left(12^{\circ}\right.$ $16^{\prime} \mathrm{N}$ lat ; 2 $09^{\prime}$ ' W long) située dans la zone climatique soudano-sahélienne du Burkina Faso (Figure 1). Le climat est du type tropical avec une saison sèche allant de novembre à avril et une saison pluvieuse de mai à octobre.

L'étude a été réalisée sous conditions pluviométriques. Les pluviométries mensuelles au cours des campagnes agricoles ont varié de 19 à 225,6 mm en 2018 et de 60,3 à 267,3 mm en 2019 (Tableau 1). Celles des dix dernières années (2010 à 2019) ont varié de 42,3 à 253,2 $\mathrm{mm}$. Les résultats indiquent des précipitations plus fortes dans les mois de juillet à septembre.

Les températures moyennes annuelles étaient de $21,7{ }^{\circ} \mathrm{C}$ en mima et $36,4{ }^{\circ} \mathrm{C}$ en 
maxima en 2018 et de $21,2^{\circ} \mathrm{C}$ en mima et de $35,3{ }^{\circ} \mathrm{C}$ en maxima en 2019 (Koumbem, 2020).

L'essai a été conduit sur un sol du type ferrugineux tropical lessivé induré présentant une carapace à environ $50 \mathrm{~cm}$ de profondeur, de texture sablo-limoneuse en surface (59,2\% de sable, $31,4 \%$ de limon et $9,4 \%$ d'argile) avec une faible capacité de rétention en eau, un $\mathrm{pH}$ (eau) de 5,4. Ces teneurs sont faibles (Koumbem, 2020) et cette faiblesse en éléments nutritifs du sol impose la nécessité donc d'améliorer sa fertilité par le travail du sol et des apports de fertilisants. L'essai a été implanté sur le même terrain pendant les deux années. Le précédent cultural du champ était du niébé cultivé pendant deux ans avant l'implantation de l'essai en 2018.

\section{Matériel végétal}

La plante test est la variété de mil IKMP5 dont le cycle végétatif est de 110 jours avec des rendements moyens de $1600 \mathrm{~kg} \mathrm{ha}^{-}$ ${ }^{1}$ en station et de $650 \mathrm{~kg} \mathrm{ha}^{-1}$ en milieu paysan. La variété IKMP5 a une bonne vigueur à la levée et est résistante à la verse, à la sécheresse, aux insectes et aux maladies (mildiou, ergot et charbon) et au striga (Comité National des Semences, 2014). Elle est beaucoup demandée par les transformateurs locaux pour la préparation des bouillies infantiles et du tô, des boissons traditionnelles comme le zom-Koom et le dolo.

\section{Fertilisants}

La matière organique utilisée est le compost présentant un $\mathrm{pH}$ de 8,1 et contenant $15,27 \%$ de carbone total (Ctot), 1,40\% d'azote total (Ntot), 2,98\% de phosphore total (Ptot), $0,64 \%$ de potassium total (Ktot) et un rapport $\mathrm{C} / \mathrm{N}$ de 10,95 . Les fertilisants minéraux utilisés sont : le NPK-S-CaO $\left(15 \mathrm{~N}-15 \mathrm{P}_{2} \mathrm{O}-15 \mathrm{KCl}+13\right.$ $\left.\mathrm{SO}_{3}+8 \mathrm{CaO}\right)$, l'urée $(30 \mathrm{~N}+3 \mathrm{MgO}+8 \mathrm{~S}+$ $0,3 \mathrm{Zn}+0,2 \mathrm{~B})$ et l'urée $(46 \mathrm{~N})$.

\section{Dispositif expérimental}

Le dispositif expérimental utilisé est un bloc complétement randomisé avec une disposition des traitements en split-plot et trois (03) répétitions où les parcelles principales correspondent à quatre (04) niveaux de travail du sol (Figure 2) et les parcelles secondaires représentées par huit (08) niveaux de fertilisation minérale avec ou sans compost (Tableau 2). La parcelle élémentaire est de dix (10) $\mathrm{m}$ de long et quatre (04) $\mathrm{m}$ de large soit 40 $\mathrm{m}^{2}$ séparée par des allées d'un $(01) \mathrm{m}$.

Les semis ont été effectués le 5 juillet en 2018 et le 23 Juillet en 2019, à une densité de $80 \mathrm{~cm}$ (entre les lignes) x $40 \mathrm{~cm}$ (entre les poquets) dans toutes les parcelles. Les démariages ont été effectués le 20 Août en 2018 et le 26 Août en 2019, à 1-2 plants par poquet dans toutes les parcelles. Le NPK a été appliqué aux poquets 15 jours après le semis et l'urée 45 jours après le semis. La technique de la microdose qui consiste à appliquer l'engrais à $3-5 \mathrm{~cm}$ du poquet de semis a été utilisée pour l'application des engrais minéraux. La dose de compost appliquée par an sur les parcelles sans zaï est de 5,0 $\mathrm{t} \mathrm{ha}^{-1}$. Sur les parcelles avec zaï, la dose traditionnellement recommandée de $300 \mathrm{~g}$ par poquet de zaï a été appliquée soit 9,4 $t \mathrm{th}^{-1}$. Le compost a été épandu à la volée dans les parcelles devant recevoir du compost avant le scarifiage, le labour et le billonnage. Pour les parcelles de zaï manuel, le compost a été appliqué dans les poquets de zaï. Pour tous les types de travail du sol, le compost a été appliqué dans les parcelles avant le semis. Les doses de compost, d'engrais minéraux NPK (encore appelé engrais coton) et d'urée appliquées sont celles recommandées (vulgarisées) au Burkina Faso par l'Institut de Recherches en Agronomie Tropicale (IRAT) et l'Institut de l'Environnement et de Recherches Agricoles (INERA) depuis des décennies et reprises par le Centre International de Développement des Engrais (IFDC) en 2018. Ces doses sont de 2,5 $\mathrm{tha}^{-1}$ par an ou 5,0 $\mathrm{tha}^{-1}$ tous les deux ans pour le compost et de $100 \mathrm{~kg}$ $\mathrm{ha}^{-1}$ de NPK plus $50 \mathrm{~kg}$ ha-1 d'urée par an pour ce qui concerne ces deux types d'engrais minéraux. Ce sont ces doses d'engrais minéraux qui ont été utilisées comme témoin dans l'étude. Les désherbages ont été faits en cas de besoin, tenant compte du niveau d'enherbement des parcelles. 
Collecte et analyse statistique des données

La récolte a été réalisée sur une superficie utile de $28,16 \mathrm{~m}^{2}$. Les données collectées ont porté sur le poids de 1000 grains, les rendements en grain et en paille. Un tableur Microsoft Excel a été utilisé pour saisir les données collectées. Les analyses statistiques ont porté sur toutes les variables mesurées et ont été effectuées à l'aide du logiciel SAS/STAT ${ }^{\circledR}$, version 9.2 (SAS Institute, 2010). Les effets ont été déclarés significatifs quand le seuil de probabilité critique était $\leq$ 0,05 .

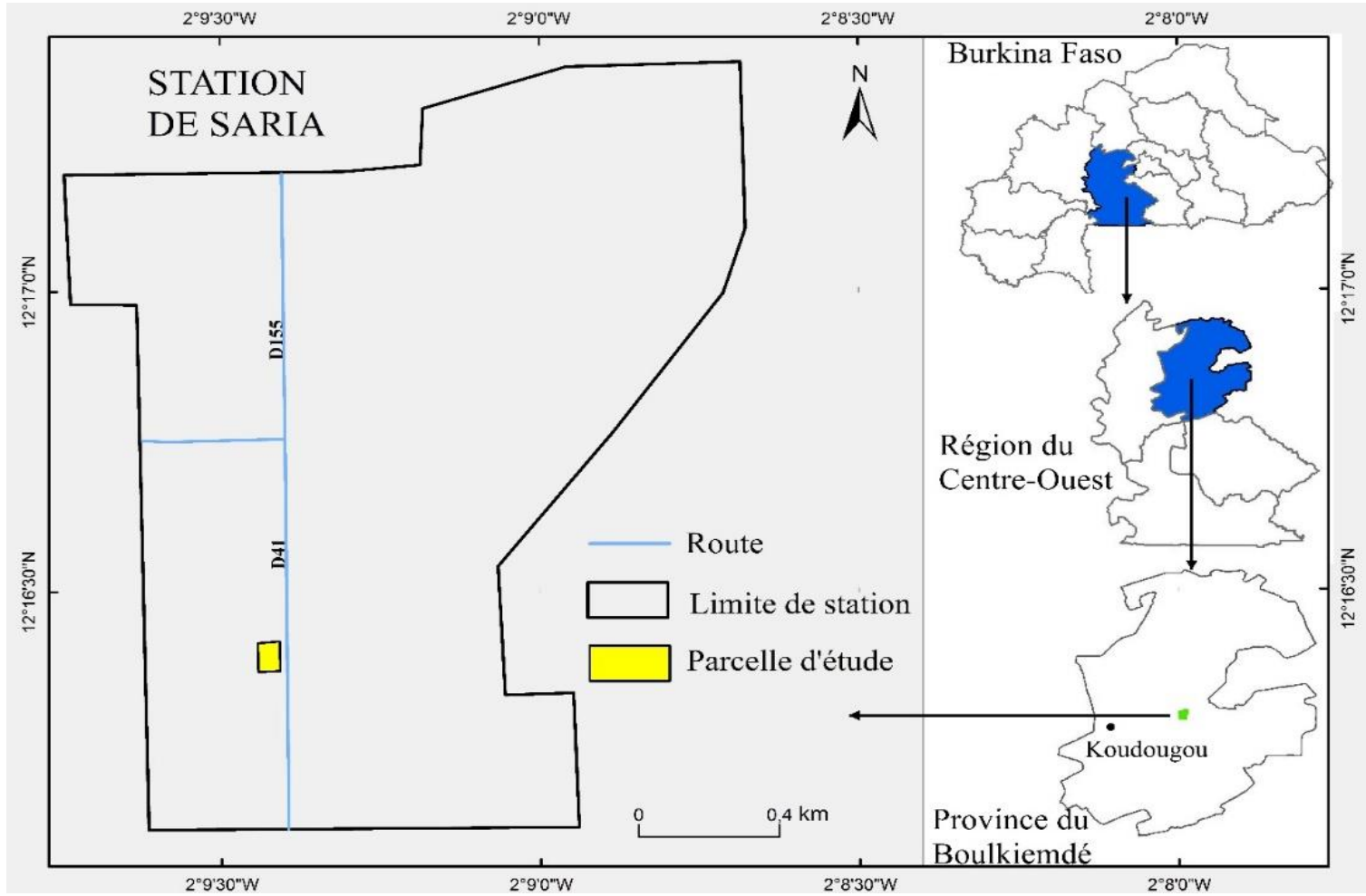

Figure 1 : Carte de localisation du site d'étude (Station de Recherches de Saria) dans la Région du Centre-Ouest.

Tableau 1 : Pluviométries des campagnes agricoles de 2018 et 2019 et la moyenne mensuelle pour la période de 2010 à 2019, Station de recherches environnementales et agricoles de Saria, Burkina Faso.

\begin{tabular}{|c|c|c|c|c|c|c|c|}
\hline \multirow{3}{*}{ Total mensuel 2018} & Mai & Juin & Juil. & Août & Sept. & Oct. & Total annuel \\
\hline & \multicolumn{7}{|c|}{ - } \\
\hline & $\begin{array}{c}51,7 \\
(5)\end{array}$ & $\begin{array}{c}99,3 \\
(9)\end{array}$ & $\begin{array}{c}190,3 \\
(16)\end{array}$ & $\begin{array}{c}210,7 \\
(15)\end{array}$ & $\begin{array}{c}225,6 \\
(14)\end{array}$ & $\begin{array}{l}19 \\
(5)\end{array}$ & $\begin{array}{c}796,6 \\
(64)\end{array}$ \\
\hline Total mensuel 2019 & $\begin{array}{c}60,3 \\
(7)\end{array}$ & $\begin{array}{c}83,5 \\
(6)\end{array}$ & $\begin{array}{c}248,2 \\
(14)\end{array}$ & $\begin{array}{c}267,3 \\
(17)\end{array}$ & $\begin{array}{c}148,5 \\
(13)\end{array}$ & $\begin{array}{l}66,7 \\
(10)\end{array}$ & $\begin{array}{c}894,5 \\
(67)\end{array}$ \\
\hline Moyenne mensuelle 2010-2019 & 63,8 & 103,8 & 198,3 & 253,2 & 159,6 & 42,3 & \\
\hline
\end{tabular}




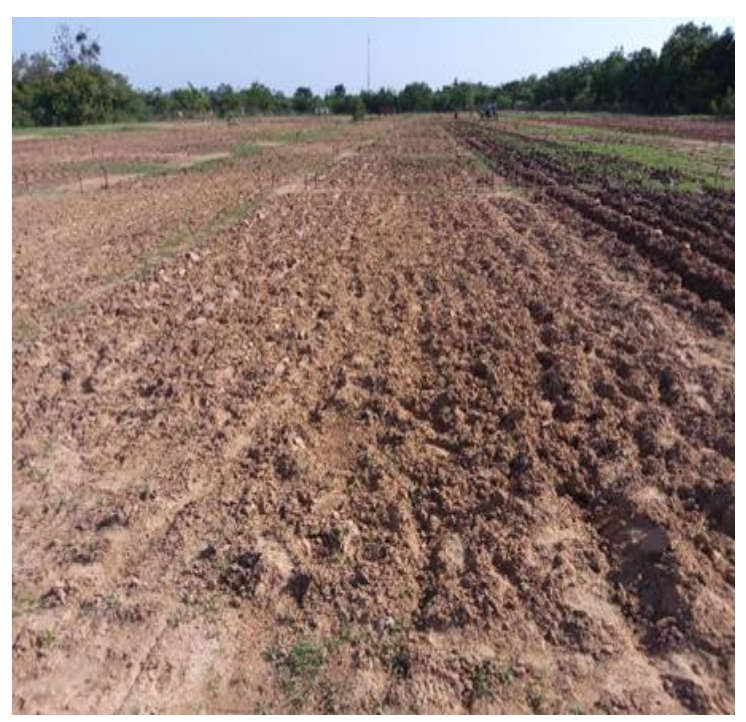

Fig.2a : Scarifiage

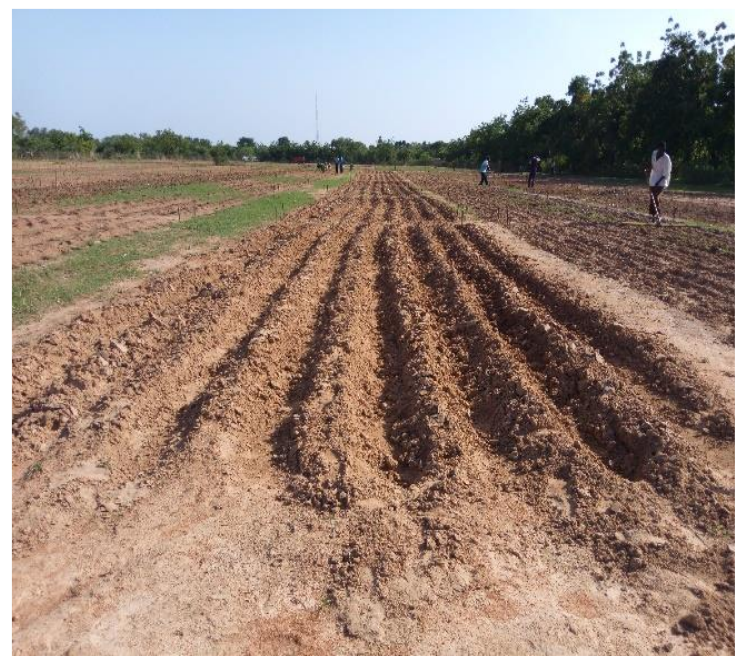

Fig.2c : Billons (sans cloisons)

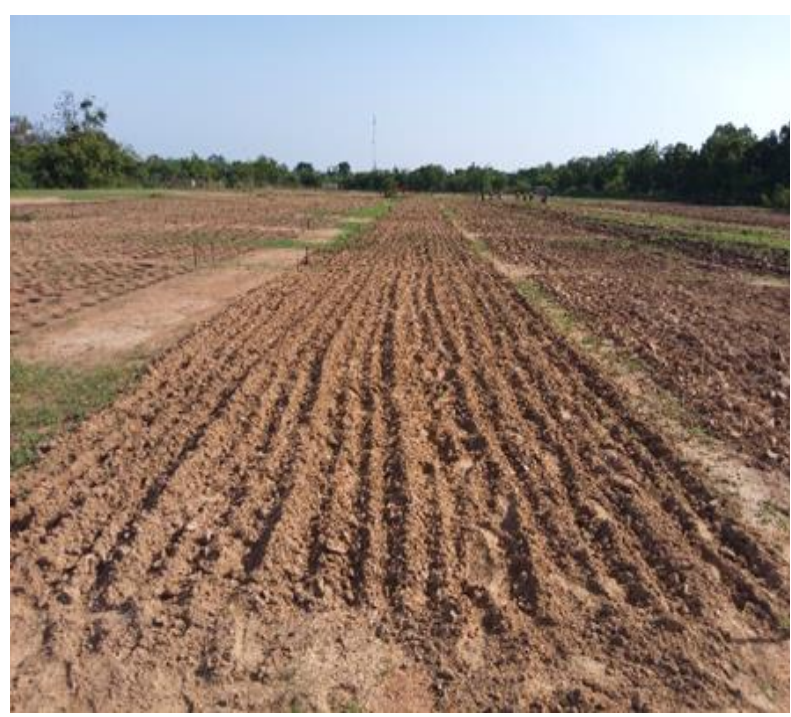

Fig.2b : Labour

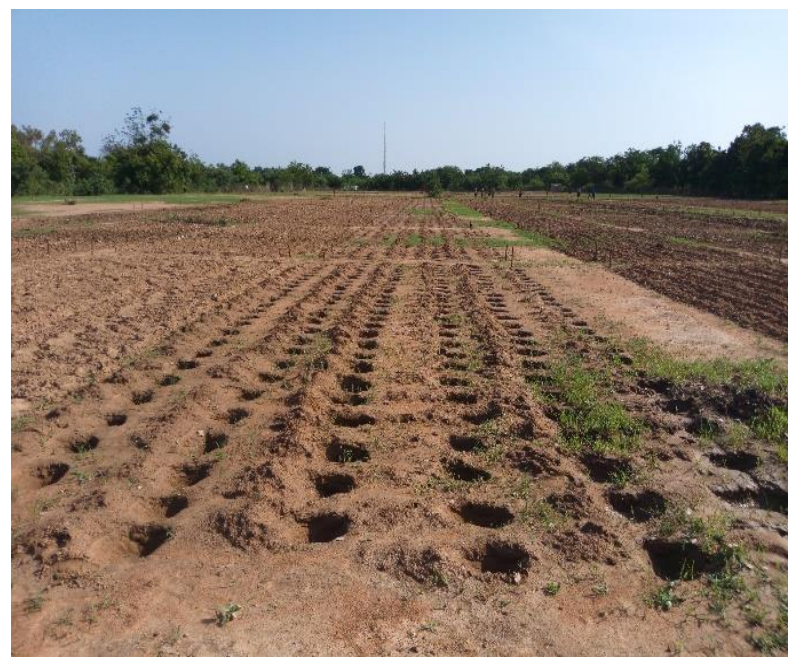

Fig.2d : Poquets de zaï manuel

Figure 2 : Les parcelles après la réalisation des opérations de travail du sol. 
Tableau 2 : Niveaux de travail du sol et de fertilisation minérale avec ou sans compost.

\section{Travail du sol (TS)}

TS1 = Scarifiage ou grattage superficiel du sol au cultivateur (houe manga) en vue d'ameublir les dix (10) premiers centimètres du sol.

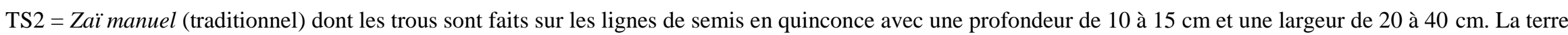
excavée est disposée en croissant en aval du trou.

TS3 = Labour à la charrue (CH9) avec les bœufs à une profondeur d'environ $15 \mathrm{~cm}$.

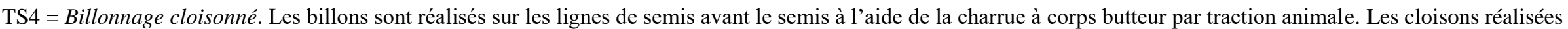
un mois après le semis à une distance de $1 \mathrm{~m}$ entre deux cloisons à l'aide de dabas, n'ont jamais été ouverts pendant les deux années.

\begin{tabular}{|c|c|c|c|c|c|c|c|c|c|}
\hline \multicolumn{10}{|l|}{ Fertilisation (F) } \\
\hline & $\mathrm{N}$ & $\mathrm{P}_{2} \mathrm{O}_{5}$ & $\mathrm{~K}_{2} \mathrm{O}$ & $\mathrm{S}$ & $\mathrm{CaO}$ & $\mathrm{Mg}$ & $\mathrm{Zn}$ & $\mathrm{B}$ & Compost \\
\hline & \multicolumn{9}{|c|}{ 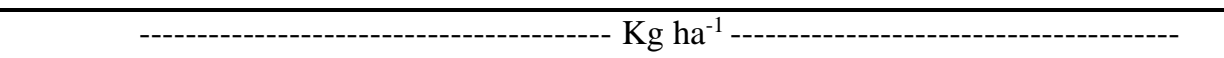 } \\
\hline $\mathrm{F} 1=100 \mathrm{~kg} \mathrm{ha}^{-1}$ de NPK-S-CaO + $50 \mathrm{~kg} \mathrm{ha}^{-1}$ d’Urée $(46 \mathrm{~N})$ & 38 & 15 & 15 & 13 & 8 & 0 & 0 & 0 & 0 \\
\hline $\mathrm{F} 2=150 \mathrm{~kg} \mathrm{ha}^{-1}$ de NPK-S-CaO + $100 \mathrm{~kg} \mathrm{ha}^{-1} \mathrm{~d}^{\prime}$ Urée $(46 \mathrm{~N})$ & 68,5 & 22,5 & 22,5 & 19,5 & 12 & 0 & 0 & 0 & 0 \\
\hline $\mathrm{F} 3=150 \mathrm{~kg} \mathrm{ha}^{-1} \mathrm{de}$ NPK-S-CaO + $100 \mathrm{~kg} \mathrm{ha}^{-1} \mathrm{~d}^{\prime}$ Urée 30 & 52,5 & 22,5 & 22,5 & 27,5 & 12 & 3 & 0,3 & 0,20 & 0 \\
\hline $\mathrm{F} 4=100 \mathrm{~kg} \mathrm{ha}^{-1}$ de NPK-S-CaO + 50 kg ha-1 d'Urée 30 & 30 & 15 & 15 & 17 & 8 & 1,5 & 0,15 & 0,10 & 0 \\
\hline $\mathrm{F} 5=\mathrm{F} 2+5 \mathrm{tha}^{-1}$ compost & 68,5 & 22,5 & 22,5 & 19,5 & 12 & 0 & 0 & 0 & 5000 \\
\hline $\mathrm{F} 6=\mathrm{F} 1+5 \mathrm{tha}^{-1}$ compost & 38 & 15 & 15 & 13 & 8 & 0 & 0 & 0 & 5000 \\
\hline $\mathrm{F} 7=\mathrm{F} 3+5 \mathrm{t} \mathrm{ha}^{-1}$ compost & 52,5 & 22,5 & 22,5 & 27,5 & 12 & 3 & 0,3 & 0,20 & 5000 \\
\hline $\mathrm{F} 8=\mathrm{F} 4+5 \mathrm{t} \mathrm{ha}^{-1}$ compost & 30 & 15 & 15 & 17 & 8 & 1,5 & 0,15 & 0,10 & 5000 \\
\hline
\end{tabular}

$\mathrm{N}$ : azote ; $\mathrm{P}_{2} \mathrm{O}_{5}$ : phosphore $; \mathrm{K}_{2} \mathrm{O}$ : potassium ; $\mathrm{S}$ : soufre ; $\mathrm{CaO}$ : calcium ; $\mathrm{Mg}$ : magnésium ; $\mathrm{Zn}$ : zinc ; $\mathrm{B}$ : bore 


\section{RESULTATS}

L'analyse des variances des facteurs testés a montré l'influence de l'effet interactif de l'année et du travail du sol sur le rendement en grain (Tableau 3) et le rendement en paille (Tableau 5). Les résultats ont également montré que ces rendements en grain et en paille ont été affectés par l'effet interactif du travail du sol et de la fertilisation (Tableaux 4 et 6). Le poids de 1000-grains quant à lui a tété uniquement affecté par l'effet principal de l'année $(\mathrm{P}=0,01)$.

\section{Variation du rendement en grain et du poids de 1000-grains du mil \\ Effet interactif de l'année et du travail du sol sur le rendement en grain}

Les résultats ont montré qu'en 2018, année de plus basse pluviométrie, le labour a engendré des rendements en grain significatifs de $1523 \mathrm{~kg} \mathrm{ha}^{-1}$ comparativement au scarifiage, billonnage cloisonné et zaï pour lesquels des rendements ont baissé respectivement de 166, 186 et $221 \mathrm{~kg} \mathrm{ha}^{-1}$ (Tableau 3). En 2019, année de plus haute pluviométrie, l'utilisation du labour a également permis des gains en rendement grain significatifs par rapport au zaï manuel (312 $\mathrm{kg} \mathrm{ha}^{-1}$ ), au scarifiage (444 kg ha$\left.{ }^{1}\right)$ et au billonnage cloisonné $\left(1087 \mathrm{~kg} \mathrm{ha}^{-1}\right)$. Les résultats ont également montré une stabilité des rendements grain du mil au cours des deux années quand le scarifiage a été utilisé, alors que les rendements variaient d'une année à l'autre pour les trois autres types de travail du sol. L'effet principal de l'année sur le poids de 1000-grains laissait observer un poids de 10,34 g en 2018 (année de plus basse pluviométrie) plus bas que celui de 2019 (année de plus haute pluviométrie) qui était de $11,91 \mathrm{~g}$.

Effet interactif du travail du sol et de la fertilisation sur le rendement en grain

Les résultats ont montré que pour tous les niveaux de travail du sol, les parcelles ayant reçu les engrais minéraux et le compost comme fertilisation organique ont permis des accroissements de rendement en grain du mil par rapport à celles qui ont reçu uniquement les engrais minéraux (Tableau 4). Ces rendements additionnels varient à l'intérieur du type de travail du sol utilisé.

Pour toutes les parcelles exception faite du billonnage cloisonné où la fertilisation F5 a permis un gain additionnel de $381 \mathrm{~kg} \mathrm{ha}^{-1}$ par rapport au témoin, les résultats ont montré des rendements plus élevés observés dans les autres parcelles particulièrement avec l'utilisation de l'engrais minéral du type F4 plus du compost (soit F8). Par rapport au témoin $\mathrm{F} 1$, le niveau de fertilisation $\mathrm{F} 8$ a engendré des rendements additionnels en grain du mil de $772 \mathrm{~kg} \mathrm{ha}^{-1}$ dans les parcelles avec le scarifiage, $738 \mathrm{~kg} \mathrm{ha}^{-1}$ ans les parcelles avec le zaï manuel et $283 \mathrm{~kg} \mathrm{ha}^{-1}$ dans les parcelles labourées. Une comparaison des rendements dus à l'application des seuls engrais minéraux par rapport à la fertilisation combinant les engrais minéraux et le compost a laissé observer des rendements additionnels dus au compost allant de 33 à $42 \%$ suivant la formule et la dose de l'engrais minéral auquel le compost a été ajouté.

\section{Variation du rendement en paille du mil Effet interactif de l'année et du travail du sol sur le rendement en paille}

Les résultats ont montré que pour l'ensemble des deux campagnes agricoles, le labour a significativement affecté le rendement en paille du mil comparativement au scarifiage, au zaï manuel et au billonnage cloisonné (Tableau 5). Cependant en 2018, année de plus basse pluviométrie, le billonnage cloisonné a favorisé l'augmentation significative du rendement en paille comparativement au labour suivi du zaï manuel et du scarifiage. En 2019, année de plus haute pluviométrie, le labour a favorisé significativement l'augmentation du rendement en paille par rapport au zaï manuel et au scarifiage suivi du billonnage cloisonné. Le labour a permis d'observer des accroissements de rendements 
en paille de $381 \mathrm{~kg} \mathrm{ha}^{-1}$ par rapport au billonnage cloisonné, de $417 \mathrm{~kg} \mathrm{ha}^{-1}$ par rapport au zaï manuel et de $601 \mathrm{~kg} \mathrm{ha}^{-1}$ par rapport au scarifiage. Les résultats ont laissé observer une variation des rendements en paille du mil d'une année à l'autre pour chacun des travaux du sol, mais en moyenne, la campagne agricole 2018 a permis d'avoir un rendement en paille plus élevé par rapport à celle de 2019.

\section{Effet interactif de la fertilisation et du travail} du sol sur le rendement en paille

Les résultats ont montré que quel que soit le travail du sol, les parcelles ayant reçu une fertilisation organo-minérale ont eu un rendement en paille élevé par rapport aux parcelles ayant reçu les engrais minéraux seuls (Tableau 6). Au niveau du zaï manuel les résultats montrent que les traitements ayant reçu du compost et de l'engrais minéral ont favorisé l'accroissement de la biomasse sèche aérienne par rapport aux traitements sans compost. Le traitement $\mathrm{CP}+150 \mathrm{~kg} \mathrm{ha}^{-1} \mathrm{de}$ NPK $+100 \mathrm{~kg} \mathrm{ha}^{-1}$ d'urée $30 \mathrm{~N}$, a augmenté le rendement en paille de $1805 \mathrm{~kg} \mathrm{ha}^{-1}$ par rapport au témoin. Dans les parcelles où le scarifiage a été réalisé, les traitements ont eu un effet similaire sur le rendement en paille. Pour les parcelles de billonnage cloisonné, le traitement $\mathrm{CP}+100 \mathrm{~kg} \mathrm{ha}^{-1} \mathrm{de}$ NPK $+50 \mathrm{~kg} \mathrm{ha}^{-1}$ d'urée $46 \mathrm{~N}$ a augmenté le rendement de $591 \mathrm{~kg} \mathrm{ha}^{-1}$ comparativement au témoin. Les résultats ont indiqué également que, pour chacun des niveaux de fertilisation organo-minérale ou minérale toute seule, le labour en général a significativement et positivement affecté le rendement paille du mil tout au long de l'expérimentation, suivi du billonnage cloisonné, du scarifiage et du zaï manuel. Une comparaison des rendements dus à l'application des seuls engrais minéraux par rapport à la fertilisation combinant les engrais minéraux et le compost a laissé observer des rendements additionnels en paille dus au compost, allant de 25 à $41 \%$ suivant la formule et la dose de l'engrais minéral auquel le compost a été ajouté.

Tableau 3 : Effet interactif de l'année (An) et du travail du sol (TS) sur le rendement en grain du mil, 2018 et 2019, Saria, Burkina Faso. [Probabilités (P) de l'analyse de variance : $\mathrm{P}_{\mathrm{An} * T S} \leq 0,01, \mathrm{P}_{\mathrm{An}}$ $\left.=0,12, \mathrm{P}_{\mathrm{TS}}=0,37\right]$.

\begin{tabular}{lccc}
\hline Travail du sol & \multicolumn{2}{c}{ Années } \\
\cline { 2 - 3 } & $\mathbf{2 0 1 8}$ & $\mathbf{2 0 1 9}$ & Moyenne \\
\cline { 2 - 3 } & $1357^{\mathrm{abA}}$ & $1480^{\mathrm{bA}}$ & $1418^{\mathrm{b}}$ \\
\cline { 2 - 3 } Scarifiage & $1302^{\mathrm{bB}}$ & $1612^{\mathrm{bA}}$ & $1457^{\mathrm{b}}$ \\
Zaï manuel & $1523^{\mathrm{aB}}$ & $1924^{\mathrm{aA}}$ & $1723^{\mathrm{a}}$ \\
Labour & $1337^{\mathrm{abA}}$ & $837^{\mathrm{cB}}$ & $1088^{\mathrm{c}}$ \\
Billonnage cloisonné & $1380^{\mathrm{A}}$ & $1463^{\mathrm{A}}$ & \\
Moyenne & &
\end{tabular}

Les valeurs suivies de la même lettre en majuscule dans une même ligne et en minuscule dans une même colonne ne sont pas significativement différentes à $\mathrm{p} \leq 0,05$. 
Tableau 4 : Effet interactif du travail du sol (TS) et de la fertilisation (F) sur le rendement en grain du mil, 2018 et 2019, Saria, Burkina Faso. [Probabilités de l'analyse de variance : $\left.\mathrm{P}_{\mathrm{TS} * \mathrm{TF}} \leq 0,01, \mathrm{P}_{\mathrm{TS}}=0,37, \mathrm{P}_{\mathrm{F}} \leq 0,01 ; \mathrm{P}_{\text {POUR LES CONTRASTES }}<0,01\right]$.

\begin{tabular}{|c|c|c|c|c|c|c|c|}
\hline \multirow[b]{2}{*}{ Fertilisation } & \multicolumn{5}{|c|}{ Travail du sol } & \multicolumn{2}{|c|}{ Contraste } \\
\hline & Scarifiage & $\begin{array}{c}\text { Zaï } \\
\text { manuel }\end{array}$ & Labour & $\begin{array}{l}\text { Billonnage } \\
\text { cloisonné }\end{array}$ & Moyenne & Type & $\begin{array}{c}\text { RA-CP } \\
(\%)\end{array}$ \\
\hline & ------- & --------- & --- $\mathrm{Kg} \mathrm{ha}^{-1}$ & 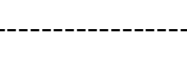 & ---- & & \\
\hline $\mathrm{F} 1=100 \mathrm{~kg} \mathrm{ha}^{-1}$ de NPK-S-CaO + $50 \mathrm{~kg} \mathrm{ha}^{-1}$ d'Urée $(46 \mathrm{~N})$ & $1030^{\mathrm{bB}}$ & $1236^{\mathrm{bAB}}$ & $1545^{\mathrm{bA}}$ & $982^{\mathrm{abB}}$ & $1198^{c}$ & $\begin{array}{l}\text { FM vs } \\
\mathrm{FM}+\mathrm{CP}\end{array}$ & - \\
\hline $\mathrm{F} 2=150 \mathrm{~kg} \mathrm{ha}^{-1}$ de NPK-S-CaO + $100 \mathrm{~kg} \mathrm{ha}^{-1} \mathrm{~d}^{\prime}$ Urée $(46 \mathrm{~N})$ & $1414^{\mathrm{abA}}$ & $761^{\mathrm{cB}}$ & $1590^{\mathrm{bA}}$ & $730^{\mathrm{bB}}$ & $1123^{\mathrm{c}}$ & F1 vs F6 & 33 \\
\hline $\mathrm{F} 3=150 \mathrm{~kg} \mathrm{ha}^{-1}$ de NPK-S-CaO $+100 \mathrm{~kg} \mathrm{ha}^{-1}$ d'Urée 30 & $1290^{\mathrm{bAB}}$ & $894^{\mathrm{bcB}}$ & $1516^{\mathrm{bA}}$ & $1136^{\mathrm{abAB}}$ & $1209^{c}$ & F2 vs F5 & 40 \\
\hline $\mathrm{F} 4=100 \mathrm{~kg} \mathrm{ha}^{-1}$ de NPK-S-CaO $+50 \mathrm{~kg} \mathrm{ha}^{-1} \mathrm{~d}^{\prime}$ Urée 30 & $1406^{\mathrm{abAB}}$ & $1024^{\mathrm{bcB}}$ & $1652^{\mathrm{bA}}$ & $901^{\mathrm{bB}}$ & $1246^{c}$ & F3 vs F7 & 42 \\
\hline $\mathrm{F} 5=\mathrm{F} 2+5 \mathrm{tha}^{-1}$ compost & $1560^{\mathrm{abB}}$ & $1742^{\mathrm{aAB}}$ & $2056^{\mathrm{aA}}$ & $1363^{\mathrm{aB}}$ & $1680^{\mathrm{ab}}$ & F4 vs F8 & 38 \\
\hline $\mathrm{F} 6=\mathrm{F} 1+5 \mathrm{tha}^{-1}$ compost & $1350^{\mathrm{bB}}$ & $1942^{\mathrm{aA}}$ & $1394^{\mathrm{bB}}$ & $1278^{\mathrm{abB}}$ & $1491^{\mathrm{b}}$ & & \\
\hline $\mathrm{F} 7=\mathrm{F} 3+5 \mathrm{tha}^{-1}$ compost & $1494^{\mathrm{abB}}$ & $2086^{\mathrm{aA}}$ & $2206^{\mathrm{aA}}$ & $1059^{\mathrm{abC}}$ & $1712^{\mathrm{a}}$ & & \\
\hline $\mathrm{F} 8=\mathrm{F} 4+5 \mathrm{tha}^{-1}$ compost & $1803^{\mathrm{aA}}$ & $1974^{\mathrm{aA}}$ & $1828^{\mathrm{abA}}$ & $1251^{\mathrm{abB}}$ & $1714^{\mathrm{a}}$ & & \\
\hline Moyenne & $1418^{\mathrm{B}}$ & $1457^{\mathrm{B}}$ & $1723^{\mathrm{A}}$ & $1087,57^{\mathrm{C}}$ & & & \\
\hline
\end{tabular}

FM : Fumure minérale ; FM+CP : Fumure minérale + Compost ; RA-CP : Rendement additionnel dû au compost. 
Tableau 5 : Effet interactif de l'année (An) et du travail du sol (TS) sur le rendement en paille du mil, 2018 et 2019, Saria, Burkina Faso. [Probabilités (P) de l'analyse de variance : $\left.\mathrm{P}_{\mathrm{An} * \mathrm{TS}} \leq 0,01, \mathrm{P}_{\mathrm{An}}=0,2, \mathrm{P}_{\mathrm{TS}}=0,75\right]$.

\begin{tabular}{|c|c|c|c|}
\hline \multirow[t]{3}{*}{ Travail du sol } & \multicolumn{2}{|c|}{ Années } & \multirow[b]{2}{*}{ Moyenne } \\
\hline & 2018 & 2019 & \\
\hline & \multicolumn{3}{|c|}{ 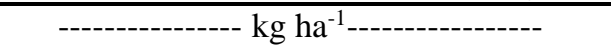 } \\
\hline Scarifiage & $3146^{\mathrm{bA}}$ & $1998^{\mathrm{bB}}$ & $2571^{\mathrm{b}}$ \\
\hline Zaï manuel & $3408^{\mathrm{bA}}$ & $2101^{\mathrm{bB}}$ & $2755^{\mathrm{b}}$ \\
\hline Labour & $3709^{\mathrm{abA}}$ & $2634^{\mathrm{aB}}$ & $3172^{\mathrm{a}}$ \\
\hline Billonnage cloisonné & $4167^{\mathrm{aA}}$ & $1415^{\mathrm{cB}}$ & $2791^{\mathrm{b}}$ \\
\hline Moyenne & $3608^{\mathrm{A}}$ & $2037^{\mathrm{B}}$ & \\
\hline
\end{tabular}

Les valeurs suivies de la même lettre en majuscule dans une même ligne et en minuscule dans une même colonne ne sont pas significativement différentes à $\mathrm{p} \leq 0,05$.

Tableau 6 : Effet interactif du travail du sol (TS) de la fertilisation (F) sur le rendement en paille du mil, 2018 et 2019, Saria, Burkina Faso. [Probabilités (P) de l'analyse de variance : $\left.\mathrm{P}_{\mathrm{TS} * \mathrm{~F}} \leq 0,01, \mathrm{P}_{\mathrm{TS}}=0,75, \mathrm{P}_{\mathrm{F}} \leq 0,01 ; \mathrm{P}_{\text {Pour les Contrastes }}<0,01\right]$.

\begin{tabular}{|c|c|c|c|c|c|c|c|}
\hline \multirow[b]{2}{*}{ Fertilisation } & \multicolumn{5}{|c|}{ Travail du sol } & \multicolumn{2}{|c|}{ Contraste } \\
\hline & Scarifiage & Zaï manuel & Labour & Billonnage cloisonné & Moyenne & Type & RA-CP (\%) \\
\hline \multicolumn{8}{|c|}{---------------------------------- Kg ha'1 --------------------------- } \\
\hline $\mathrm{F} 1=100 \mathrm{~kg} \mathrm{ha}^{-1}$ de NPK-S-CaO + $50 \mathrm{~kg} \mathrm{ha}^{-1}$ d'Urée $(46 \mathrm{~N})$ & $2170^{\mathrm{aB}}$ & $2289^{\mathrm{bAB}}$ & $3211^{\mathrm{abA}}$ & $2747^{\mathrm{abAB}}$ & $2604^{\mathrm{b}}$ & $\begin{array}{c}\text { FM vs } \\
\text { FM+CP }\end{array}$ & - \\
\hline $\mathrm{F} 2=150 \mathrm{~kg} \mathrm{ha}^{-1}$ de NPK-S-CaO + $100 \mathrm{~kg} \mathrm{ha}^{-1} \mathrm{~d}^{\prime}$ Urée $(46 \mathrm{~N})$ & $2469^{\mathrm{aA}}$ & $1272^{\mathrm{cB}}$ & $2920^{\mathrm{abA}}$ & $2000^{\mathrm{bAB}}$ & $2165^{\mathrm{b}}$ & F1 vs F6 & 40 \\
\hline F3 $=150 \mathrm{~kg} \mathrm{ha}^{-1}$ de NPK-S-CaO + $100 \mathrm{~kg} \mathrm{ha}^{-1}$ d'Urée 30 & $2788^{\mathrm{aA}}$ & $1792^{\mathrm{bcB}}$ & $2862^{\mathrm{bA}}$ & $3089^{\mathrm{aA}}$ & $2632^{b}$ & F2 vs F5 & 28 \\
\hline $\mathrm{F} 4=100 \mathrm{~kg} \mathrm{ha}^{-1}$ de NPK-S-CaO + 50 kg ha-1 d'Urée 30 & $2376^{\mathrm{aA}}$ & $1828^{\mathrm{bcB}}$ & $3156^{\mathrm{abA}}$ & $1823^{\mathrm{bA}}$ & $2296^{\mathrm{b}}$ & F3 vs F7 & 25 \\
\hline $\mathrm{F} 5=\mathrm{F} 2+5 \mathrm{t} \mathrm{ha}^{-1}$ compost & $2709^{\mathrm{aB}}$ & $3820^{\mathrm{aA}}$ & $3442^{\mathrm{abAB}}$ & $3337^{\mathrm{aAB}}$ & $3327^{\mathrm{a}}$ & F4 vs F8 & 41 \\
\hline $\mathrm{F} 6=\mathrm{F} 1+5 \mathrm{t} \mathrm{ha}^{-1}$ compost & $2626^{\mathrm{aA}}$ & $3427^{\mathrm{aA}}$ & $2836^{\mathrm{bA}}$ & $3197^{\mathrm{aA}}$ & $3021^{\mathrm{ab}}$ & & \\
\hline $\mathrm{F} 7=\mathrm{F} 3+5 \mathrm{t} \mathrm{ha}^{-1}$ compost & $2367^{\mathrm{aB}}$ & $4094^{\mathrm{aA}}$ & $3852^{\mathrm{aA}}$ & $2843^{\mathrm{abB}}$ & $3289^{\mathrm{a}}$ & & \\
\hline $\mathrm{F} 8=\mathrm{F} 4+5 \mathrm{t} \mathrm{ha}^{-1}$ compost & $3071^{\mathrm{aA}}$ & $3516^{\mathrm{aA}}$ & $3095^{\mathrm{abA}}$ & $3291^{\mathrm{aA}}$ & $3243^{a}$ & & \\
\hline Moyenne & $2571^{\mathrm{B}}$ & $2755^{\mathrm{B}}$ & $3172^{\mathrm{A}}$ & $2791^{\mathrm{B}}$ & & & \\
\hline
\end{tabular}

Note : Les valeurs suivies de la même lettre en majuscule dans une même ligne et en minuscule dans une même colo 


\section{DISCUSSION}

\section{Effets des traitements sur le rendement en grain du mil}

L'application des engrais minéraux associés avec le compost a significativement augmenté le rendement en grains du mil comparativement à la fertilisation minérale seule $\left(100 \mathrm{~kg} \mathrm{ha}^{-1}\right.$ de NPK $+50 \mathrm{~kg} \mathrm{ha}^{-1}$ d'urée $46 \mathrm{~N}$ soit F1, $150 \mathrm{~kg} \mathrm{ha}^{-1}$ de NPK + $100 \mathrm{~kg} \mathrm{ha}^{-}$ ${ }^{1}$ d'urée $46 \mathrm{~N}$ soit F2, $150 \mathrm{~kg} \mathrm{ha}^{-1}$ de NPK + $100 \mathrm{~kg} \mathrm{ha}^{-1}$ d'urée $30 \mathrm{~N}$ soit $\mathrm{F} 3,100 \mathrm{~kg} \mathrm{ha}^{-1} \mathrm{de}$ $\mathrm{NPK}+50 \mathrm{~kg} \mathrm{ha}^{-1}$ d'urée $30 \mathrm{~N}$ soit F4). Les résultats ont montré que l'ajout du compost aux différentes doses d'engrais considérés dans l'étude ont permis des gains additionnels en grain allant de 33 à 42\%. La fertilisation organo-minérale aurait donc apporté les éléments nutritifs nécessaires au développement et à la production du mil avec des accroissements en rendements du mil comme l'avait indiqué Maman et al. (2000). L'application des engrais minéraux est une source d'éléments nutritifs utilisables immédiatement par la plante pour son alimentation comme l'avaient indiqué Hien (2004), Dutordoir (2006) et Efthimiadou et al. (2010). Quand ces engrais minéraux sont associés aux matières organiques comme le compost (jouant un rôle amendant), l'assimilation des éléments nutritifs qu'ils apportent est encore plus favorisée (Hien, 2004 ; Dutordoir, 2006 ; Efthimiadou et al., 2010).

Le compost quant à lui aurait amélioré les propriétés physiques, chimiques et biologiques du sol et alimenté de façon progressive la plante par la libération des éléments nutritifs. Hien (2004) a relevé le rôle bénéfique des fumures organo-minérales dans l'amélioration du statut organo-minéral du sol et de son interaction probable sur les propriétés physiques du sol dont la densité apparente, la porosité et l'état hydrique. Il a montré que l'apport de matières organiques sous forme de fumier, de composts ou de pailles en association avec de l'engrais minéral, notamment $\mathrm{N}$, permettait un accroissement simultané de la productivité et la stabilité interannuelle des rendements. Il note par ailleurs que la fertilisation par des engrais minéraux en association avec la fumure organique améliore plus la production de façon substantielle tout en assurant une meilleure protection des sols contre la baisse de la fertilité chimique. Des résultats similaires ont été obtenus par Zeinabou et al. (2014) sur un sol sableux. Plusieurs travaux de recherche conduits sur le même type de sol que celui utilisé dans la présente étude ont montré le rôle bénéfique de la fertilisation organo-minérale sur les rendements des cultures céréalières (Sawadogo et al., 2008 ; Palé, 2012 ; Zeinabou et al., 2014 ; Dabré et al., 2016). Dans les sols compactés structurellement inertes, le labour a montré son effet bénéfique en ce sens qu'il permet de réduire la densité apparente du sol, d'accroitre la porosité totale ainsi que la proportion des macropores, d'augmenter la capacité de rétention en eau du sol et enfin d'améliorer le développement du système racinaire du mil et partant sa productivité (Hien, 2004 ; Valet et al., 2007). Fatondji et al. (2001) ont rapporté des rendements additionnels de $500 \mathrm{~kg}$ engendrés grâce à la technique du zaï combinant l'eau et les nutriments dans les poquets dans le Sahel et particulièrement sur des sols sablonneux. Zombré (2003) et Zougmoré et al. (2004) ont également obtenu des accroissements de rendements du fait de l'utilisation du zaï. Les résultats de la présente investigation ont par ailleurs montré le rôle bénéfique du labour dans la production du grain du mil, en ce sens qu'il a permis des accroissements substantiels en rendements en grain de 266 à $635 \mathrm{~kg} \mathrm{ha}^{-1}$ par rapport aux autres travaux du sol. Par ailleurs, les rendements du mil dans les parcelles de billonnage cloisonné en année de basse pluviométrie étaient plus élevés qu'en année de haute pluviométrie. Cette variation serait probablement due aux stagnations d'eau dans certaines parcelles de billonnage cloisonné en 2019.

Des résultats obtenus par Fatondji et al. (2001) et ceux de la présente étude montrent que la réponse du mil à l'utilisation d'un travail du sol donné dépend de la localité où l'étude a été conduite et partant du type de sol sur lequel l'expérimentation a été réalisée. 


\section{Effets des traitements sur le rendement en paille du mil}

Les résultats ont montré que l'ajout du compost aux différentes doses d'engrais minéraux utilisés dans l'étude ont permis des gains additionnels en paille allant de 25 à $41 \%$. Comme dans le cas du grain, pour la production de la paille, la fertilisation organo-minérale aurait apporté les éléments nutritifs nécessaires au développement et à la production du mil avec des accroissements en rendements (Maman et al., 2000). Les engrais minéraux sont une source d'éléments nutritifs utilisables immédiatement par la plante pour son alimentation et quand ils sont associés aux matières organiques comme le compost jouant un rôle amendant, l'assimilation des éléments nutritifs apportés par les engrais minéraux est encore favorisée (Hien, 2004; Dutordoir, 2006 ; Efthimiadou et al., 2010) d'où une augmentation de la production en paille. Comme l'ont démontré Fatondji et al. (2001), Ouédraogo et al. (2001) et Palé et al. (2009), l'application du compost a généralement permis un accroissement des rendements par sa lente libération des nutriments aux stades critiques de croissance des plants.

Les résultats ont également montré le rôle bénéfique du labour dans la production de paille du mil avec des accroissements substantiels des rendements allant de 381 à 601 $\mathrm{kg} \mathrm{ha}{ }^{-1}$. Des résultats similaires ont été rapportés par Hien (2004).

\section{Conclusion}

L'étude a été conduite dans la station de recherche agronomique de Saria, en Zone soudano-sahélienne. Les effets du travail du sol et de la fertilisation organo-minérale sur la variété IKMP5 du mil ont été étudiés afin d'identifier des technologies de gestion de l'eau et de la fertilisation organo-minérale qui permettent d'optimiser le rendement du mil. Les rendements en grain ont été affectés par l'effet du travail du sol et de la fertilisation ainsi que par leur effet interactif. En effet, l'étude a montré que la fertilisation minérale en combinaison avec la fertilisation organique comme le compost a permis des gains substantiels en rendements allant de 33 à $42 \%$ pour le grain et de 25 à $41 \%$ pour la paille, comparativement à l'application de l'engrais minéral seul. Pour ce qui est du travail du sol, il a été clairement démontré que le labour avec son effet bénéfique d'amélioration de la structure du sol et du développement du système racinaire de la plante et partant de sa productivité, a été le meilleur travail du sol au cours de la présente étude. Sur la base de ces résultats, les investigateurs recommandent l'utilisation du labour comme travail du sol et l'application des engrais minéraux, particulièrement la dose de $100 \mathrm{~kg}$ ha- $1 \mathrm{de}$ NPK-S-CaO + $50 \mathrm{~kg}$ ha- 1 d'Urée $30 \mathrm{~N}$ en combinaison avec le compost (soit F8) pour une optimisation des rendements du mil dans la Zone soudano-sahélienne du Burkina Faso.

\section{CONFLIT D'INTERETS}

Les auteurs déclarent qu'ils n'ont aucun conflit d'intérêts concernant cet article.

\section{CONTRIBUTIONS DES AUTEURS}

Tous les auteurs ont contribué d'une manière égalitaire à la réalisation de l'étude et à la rédaction du présent article et sont unanimes quant à la soumission du manuscrit dans ce journal pour sa publication.

\section{REMERCIEMENTS}

Les auteurs remercient l'Institut de l'Environnement et de Recherches Agricoles (INERA) du Burkina Faso et le Programme d'Amélioration de la Productivité Agricole des Petits Exploitants pour l'Afrique Subsaharienne (SAPEP) pour leurs supports administratifs, financiers et matériels pour la réalisation de l'étude.

\section{REFERENCES}

Békoye MB. 2011. Caractérisation chimique et minérale des grains de mil [Pennisetum glaucum (L.) R. Br.] de Côte d'Ivoire. Int. J. Biol. Chem. Sci., 5(5): 2039-2044. DOI: $10.4314 / \mathrm{ijbcs.v5i5.25}$

Comité National des Semences. 2014. Catalogue national des espèces et variétés agricoles du Burkina Faso. CNS, Ouagadougou, Burkina Faso, 81p. 
Dabré A, Hien E, Somé D, Drevon JJ. 2016. Impacts des pratiques culturales sur la production du sorgho (Sorghum bicolor L.) et du niébé (Vigna unguiculata (L.) Walp.) et sur le bilan partiel de l'azote sous niébé au Burkina Faso. Int. J. Biol. Chem. Sci., 10(5): 2215-2230. http://dx.doi.org/10.4314/ijbcs.v10i5.22.

Diouf O. 2001. Réponses agro-physiologiques du mil (Pennisetum glaucum (L.) R. Br) à la sècheresse, influence de la nutrition azotée. Thèse de Doctorat en sciences, Université libre de Bruxelles, $160 \mathrm{p}$.

Dutordoir CD. 2006. Impact de pratiques de gestion de la fertilité sur les rendements en mil dans le Fakara (Niger). Mémoire de Bio-Ingénieur, Faculté d'ingénierie biologique, agronomique et environnementale, Université Catholique de Louvain, p. 33-140.

Eftimladou A, Bilalis D, Karkanis A, FroudWilliams B. 2010. Combined organic/inorganic fertilization enhance soil quality and increased yield, photosynthesis and sustainability of sweet maize crop. Aust. J. Crop Sci., 4(9): 722-729.

Fatondji D, Martius C, Vlek, P. 2001. Zaï - A traditional technique for land rehabilitation in Niger. ZEFnews, 8: 1-2.

Hien E. 2004. Dynamique du carbone dans un Acrisol ferrique du Centre-Ouest Burkina : Influence des pratiques culturales sur le stock et la qualité de la matière organique. Thèse de doctorat en Sciences des Sol, Ecole Nationale Supérieure Agronomique de Montpellier, France, $138 \mathrm{p}$.

IFDC. 2018. Recommandations 2018 d'engrais pour l'Afrique de l'Ouest. IFDC, $42 \mathrm{p}$.

Kohio NE, Touré GA, Sedogo PM, Ambouta KJ-M. 2017. Contraintes à l'adoption des bonnes pratiques de Gestion Durable des Terres dans les zones soudaniennes et soudano-sahéliennes du Burkina Faso. Int. J. Biol. Chem. Sci., 11(6): 2982-2989. DOI: $10.4314 /$ ijbcs.v11i6.34.

Koumbem M. 2020. Effets du travail du sol et de la fertilisation organo-minérale sur les rendements du mil et la quantité de quelques éléments exportés par la plante dans la région centre du Burkina Faso. Mémoire de Mater en Géoressources/Sols et Environnement, Université Joseph KIZERBO, Ouagadougou, Burkina Faso, 62 p.

MAAH (Ministère de l'Agriculture et des Aménagements Hydro-agricoles). 2017a. Rapport général des résultats définitif de la campagne agricole 2016/2017 et des perspectives de la situation alimentaire et nutritionnelle. MAAH, Ouagadougou, Burkina Faso, p. 7-17.

MAAH (Ministère de l'Agriculture et des Aménagements Hydro-agricoles). 2017b. Plan stratégique pour les statistiques agricoles et rurales du Burkina Faso, 2016-2020 (PSSAR_BF 2016-2020). MAAH, Ouagadougou, Burkina Faso, p. 45-70.

Nacro S, Ouédraogo S, Traoré K, Sankara E, Kaboré C, Ouattara B. 2010. Effets comparés des pratiques paysannes et des bonnes pratiques agricoles de gestion de la fertilité des sols sur les propriétés des sols et les rendements des cultures dans la zone sud soudanienne du Burkina Faso. Int. J. Biol. Chem. Sci., 4(4): 1044-1055. DOI: 10.4314/ijbcs.v4i4.63042

Ouédraogo E, Mando A, Zombré NP. 2001. Use of compost to improve soil properties and crop productivity under low input agricultural system. West Africa Agric. Ecosyst. Environ., 84(3): 259-266. DOI: https://doi.org/10.1016/S01678809(00)00246-2.

Palé S, Mason SC, Taonda SJ-B. 2009. Water and fertilizer influence on yield of grain sorghum varieties produced in Burkina Faso. S. Afr. J. Plant Soil, 26(2): 91- 97. DOI:

https://doi.org/10.1080/02571862.2009.1 0639939.

Palé S. 2012. Quantitative and qualitative studies on grain sorghum for traditional beer (dolo) production in Burkina Faso. Thesis of PhD in Agronomy, University of KwaZulu-Natal, Republic of South Africa, $150 \mathrm{p}$. 
SAS Institute. 2010. SAS/STAT ${ }^{\circledR}$, version 9.2. SAS Institute, Cary, North Carolina.

Sawadogo H, Bock L, Lacroix D, Zombré NP. 2008. Restauration des potentialités de sols dégradés à l'aide du zaï et du compost dans le Yatenga (Burkina Faso). Biotechnol. Agron. Soc. Environ., 12(3): 279-290.

Sermé I. 2014. Growth and yield of sorghum under different conservation tillage and water and nutrient management practices in the South Sudan zone of Burkina Faso. PhD Thesis, Department of Crop and Soil Sciences, Faculty of Agriculture, p. 9095.

Traoré B. 2009. Effets des techniques de gestion de la fertilité sur le sol et sur les systèmes de culture à base de mil dans la région de Mopti au Mali. Thèse de doctorat en Ecologie Appliquée, Université de Bamako, Mali. 142 p.

Valet S, Motélica-Heino M, Le Coustumer PH. 2007. Influence géomorphologique et structurale des sols sur le report hydrique : Conséquences sur le fonctionnement hydrique des sols et sur la biomasse en zone soudano-sahélienne. $32^{\text {eme }}$ Journée Scientifique du GFHN "De la particule aux milieux poreux : Formation, évolution \& transferts", Nantes, France, 4 p.

Zeinabou H, Mahamane S, Bismarck BN, Bado BV, Lompo L, Bationo A. 2014. Effet de la combinaison des fumures organo- minérales et de la rotation niébé-mil sur la nutrition azotée et les rendements du mil au sahel. Int. J. Biol. Chem. Sci., 8(4): 1620-1632. DOI: 10.4314/ijbcs.v8i4.24.

Zombré NP. 2003. Les sols très dégradés (Zipella) du Centre Nord du Burkina Faso : Dynamique, Caractéristiques morphobio-pédologiques et impacts des techniques de restauration. Thèse de Doctorat ès Sciences Naturelles, Université de Ouagadougou, Burkina Faso.

Zongo KF. 2013. Associations légumineuses céréales dans les agrosystèmes soudano sahéliennes du Burkina Faso : Perceptions et pratiques paysannes, effets du zaï et des amendements organiques et organo-minéraux sur les rendements des cultures associées niébé-sorgho. Mémoire de DEA en Science du sol, IDR, Université Polytechnique de Bobo, Burkina Faso, $57 \mathrm{p}$.

Zougmoré BR. 2003. Integrated water and nutrient management for sorghum production ln semi-arid Burkina Faso. Ph.D Thesis, Wageningen University, Netherlands, $205 \mathrm{p}$.

Zougmoré BR, Mando A, Stroosnijder L, Guillobez S. 2004. Nitrogen flows and balances as affected by soil water and nutrients management in semi-arid Burkina Faso. Field Crops Res., 90:235244. DOI:10.1016/j.fcr.2004.03.006 